\title{
An illustrated taxonomic key to genera of Mirinae (Hemiptera: Heteroptera: Miridae) with three new records from Iran
}

\author{
Илмюстрированный определитель родов Mirinae (Hemiptera: \\ Heteroptera: Miridae) и три новых находки дмя Ирана
}

\author{
Mozhgan Zamani, Reza Hosseini* \\ М. Замани, Р. Хоссейни
}

\begin{abstract}
Department of Plant Protection, Faculty of Agricultural Sciences, University of Guilan, Rasht, Iran. P.O. Box: 41635-1314. E-mail: rhosseini@guilan.ac.ir, r_hosseini@yahoo.com

*corresponding author
\end{abstract}

KEY WORDS: Identification key, female genitalia, New record, Mirinae, Miridae, Iran.

КЛЮЧЕВЫЕ СЛОВА. Определитель, гениталии самок, новые находки, Mirinae, Miridae, Иран.

ABSTRACT. A comprehensive identification key has been compiled for 35 genera belonging to three tribes of the Mirinae subfamily found in Iran. Illustration of male and female genitalia for most genera, digital images of habitus and other diagnostic characters were provided to aid unequivocal identification. Rauniella ishtar (Linnavuori, 1984), Cyphodema humbaba Linnavuori, 1984, and Stenotus binotatus (Fabricius, 1794) are briefly redescribed and reported as three new species for Iranian fauna.

РЕЗЮМЕ. Составлен определитель для 35 родов, принадлежащих к трем трибам подсемейства Mirinae, известных из Ирана. Приведены изображения габитуса, мужских и женских гениталий и других диагностических признаков для большинства родов, позволяющие однозначно идентифицировать эти роды. Rauniella ishtar (Linnavuori, 1984), Cyphodema humbaba Linnavuori, 1984 и Stenotus binotatus (Fabricius, 1794) кратко переописаны и указаны как новые виды для фауны Ирана.

\section{Introduction}

Miridae or plant bugs are the largest family of Hemiptera, with more than 11020 described species in the world [Cassis, Schuh, 2012]. This family comprises seven subfamilies including Deraeocorinae, Mirinae, Orthotylinae, Cylapinae, Bryocorinae, Isometopinae, and Phylinae. Among them, Mirinae with at least 4100 species in approximately 350 genera is considered to be one of the most diverse and complex group of Miridae. Most species inhabit on grasses and herbaceous plants and appear to be phytophagous [Schuh, Slater, 1995; Wheeler, 2001]. Mirinae subfamily has been categorized into six tribes including Herdoniini, Hyalopeplini, Mecistoscelini, Mirini, Resthenini, and Stenodemini [Schwartz, 2008; Cassis, Schuh, 2012]. Three of them, namely Herdoniini, Mirini and Stenodemini have been reported from Iran [Ghahari, Cherot, 2014]. These tribes respectively comprise with 38,250 and 34 genera in the world [Schuh, 2002-2013], where about 1, 27 and 6 genera found and documented from Iran [Aukema, Rieger, 1999; Aukema et al., 2013]. Validity of report for Cyphodema mendosa Montandon, 1887 is in doubt and it is unclear if vouchers were deposited for further examination. The genus Hadrodemus was also erroneously recorded from Iranian fauna [Aukema et al., 2013].

Although the Mirinae is one of the best studied subfamilies of Miridae in the world from a taxonomic perspective, there are few valid keys designed specially to aid the identification of genera in this group [Carvalho, 1955; Kerzhner, 1964; Wagner, Weber, 1964; Wagner, 1974; Rosenzweig, 2001; Schwartz, 2008]. So far only a few taxonomic works have been done on the subfamily in Iran [e.g., Linnavouri, 1999, 2006, 2009; Hosseini, 2014, $2015,2016]$ and no appropriate taxonomic key has been compiled for identification of Iranian genera.

During spring-summer of 2018 we conducted a field investigation in mountainous regions and deep valleys in Guilan, Mazandaran and western part of Kurdistan provinces. Examination of sampled material resulted in finding of three species new to Iranian fauna which are briefly redescribed below.

We also compiled a comprehensive taxonomic key for identification of all Mirinae genera currently known from Iran. Illustration of male and female genitalia of

How to cite this article: Zamani M., Hosseini R. 2020. An illustrated taxonomic key to genera of Mirinae (Hemiptera: Heteroptera: Miridae) with three new records from Iran // Russian Entomol. J. Vol.29. No.1. P.20 32. doi: 10.15298/rusentj.29.1.04 
most of genera, digital images of habitus and diagnostic characters for new record species are provided to aid unequivocal identification.

\section{Materials and methods}

In an ongoing faunal study in Iran, mirid specimens were collected by sweeping during spring-summer 2018 from Guilan, Mazandaran and also the western part of Kurdistan provinces. Authentically identified species available in the insect collection of the Natural History Museum of the University of Guilan (UGNHM) were used for compiling generic taxonomic key, although, Capsus, Pantilius and Rhabdomiris were not available for examination. Adult specimens were examined using a stereomicroscope (GX Microscope, Australia). All measurements were made with ocular micrometer and are given in millimeters. Dissections of male genitalia were performed using the technique described by Scudder and Schwartz [2012]. The male and female genitalia were photographed with a Canon EOS 600D DSLR camera attached to Leica DM 1000 microscope and illustrated using a drawing tube attached to an $\mathrm{BH}-2$ Olympus microscope. Color images of the adult drymounted specimens were captured using a hand-made automated digital imaging system composed of a Canon EOS 70D DSLR camera, Canon EF $100 \mathrm{~mm} \mathrm{f/2.8} \mathrm{USM}$ Macro lens and a $65 \mathrm{~mm}$ Meike macro extension tube. Partially focused images (more than 65) were combined using Helicon Focus image stacking software (ver. 6) (http://www.heliconsoft.com). Images were edited using Adobe Photoshop CC 2017. Newly collected and examined specimens are deposited in the insect collection of the Natural History Museum of the University of Guilan (UGNHM), Rasht, Iran. Terminology of the male and female genitalia follows Konstantinov [2003] and Schwartz [2008] respectively.

\section{Taxonomy}

Cyphodema Fieber, 1858

Cyphodema humbaba Linnavuori, 1984

Figs 3-4, 7-9, 81 .

SPECIMENS EXAMINED. Iran, Kurdistan province: Sarvabad; Daraki $20^{\prime}, 2$, $\left(35^{\circ} 19^{\prime} \mathrm{N} 46^{\circ} 09^{\prime} \mathrm{E}, 1831 \mathrm{~m}\right)$, S. Mohammadi, sweeping. 15.VI.2018.

DIAGNOSIS. Cyphodema humbaba is recognized by the following characters: interocular width/width of eye $\sigma^{\top} / q^{\circ}$ : 1.38-1.5/ 1.6; labium reaching or slightly surpassing metacoxa; clavus blackish, posteriorly stramineous; corium posteriorly with large blackish marking; tibia with golden spines; pygophore with a prominent denticle (Fig. 3); sensory lobe of left paramere dentate; right paramere basally dentate; vesica with a long spiculum; sclerotized rings of female genitalia small and oval; dorsal labiate plate wide and laterally prominent; dorsal structure of posterior wall disk-shaped and spinose.

MEASUREMENTS. Body length $\sigma^{7} / 0$ : 5.2-5.5/ 4.85.2; width $\sigma^{\top} /$ : $: 2.13 / 2.2$; interocular width/width of eye $\sigma^{\top} /$ ๆ: 1.38-1.5/ 1.6; lengths of antennal segments I-IV $\sigma^{\top} /{ }^{\circ}$ $0.5-0.61,1.6,1,0.8 / 0.5,1.44-1.55,0.96-1.07,0.84-0.87$; 2nd segment $\sigma^{7} / 9$ : $1.6 \mathrm{x} / 1.4-1.5 \mathrm{x}$ as long as width of head; length of pronotum (dorsal view) $\sigma^{\top} / 9$ : 0.96-0.98/ 0.941.07; width of posterior margin of pronotum $\sigma^{7} / 9: 1.7 / 1.83$.

MALE GENITALIA. Left paramere sickle-shaped, lateral side of sensory lobe with setiferous tubercles transformed into distinct teeth (Fig. 8); right paramere broad and short, basal margin dentate, ventrally with small setiferous tubercles (Fig. 9); vesica with several membranous lobes, a single spiculum and a small spinulated plate near secondary gonopore, one of the main membranous lobes laterally with small dentate field, another one dorsally dentate and sclerotized, secondary gonopore large and oval (Fig. 7).

FEMALE GENITALIA. Dorsal labiate plate wide, laterally prominent, with pointed and reinforced margins; sclerotized rings small, oval, distinctly separated, placed laterally, obliquely oriented; posterior wall with large interramal sclerites (medially fused), small and spinose interramal lobes (medially separated), broadly rounded, disk-shaped and spinose dorsal structure and prominent medial process (Fig. 81).

COMMENTS. Nine species of Cyphodema have been described in the world [Schuh, 2002-2013], and Cyphodema humbaba is the first record from Iran. Collected on herbaceous host plants in meadow.

\section{Rauniella Rosenzweig, 1997}

\section{Rauniella ishtar (Linnavuori, 1984)}

Figs 5-6, 10-13, 89.

SPECIMENS EXAMINED. Iran, Kurdistan province: Sarvabad; Daraki $50^{\prime}$, 1 우 $\left(35^{\circ} 19^{\prime} \mathrm{N} 46^{\circ} 09^{\prime} \mathrm{E}, 1831 \mathrm{~m}\right)$, Bahram abad $\left(35^{\circ} 21^{\prime} \mathrm{N} 46^{\circ} 14^{\prime} \mathrm{E}, 1183 \mathrm{~m}\right) \mathrm{S}$. Mohammadi, sweeping. 15.VI.2018.

DIAGNOSIS. Rauniella ishtar can be distinguished by the following characters: interocular width/width of eye $\sigma^{7} / 9$ : 1.4-1.57/ 1.73; pronotum laterally below the humeral angles with black markings; femora with rows of dark brown spots; tibia with black spines; lateral side of left paramere subapically with a large dentate projection; right paramere medially constricted; vesica without spiculum; secondary gonopore forming an inverted saddle; sclerotized rings of female genitalia large, elongate-oval; dorsal labiate plate with a large antero-medial projection; median process of posterior wall prominent.

MEASUREMENTS. Body length $\sigma^{7} / 9$ : 7.75-8.15/ 7.89; width $\sigma^{7} / 9$ : 2.41/ 2.62; interocular width/width of eye $\sigma^{7} / 9$ : 1.4-1.57/ 1.73; lengths of antennal segments I-IV $\sigma^{7} /$ P : 1.16$1.23,2.86-3.16,1.83-1.94, ? / 1.07,2.98,1.6, ? ; 2^{\text {nd }}$ segment $\sigma^{7} /$ : $: 2.4-2.7 \mathrm{x} / 2.3 \mathrm{x}$ longer than width of head; length of pronotum (dorsal view) $\sigma^{\top} / O: 1.19 / 1.37$; width of posterior margin of pronotum $\sigma^{7} /$ : $: 2-2.15 / 2.36$.

MALE GENITALIA. Apophysis of left paramere forming almost right angle with its body, with large subapical dentate projection on lateral side, apically curved sideward, sensory lobe and lateral margin of left paramere with setiferous tubercles transformed into distinct teeth (Figs 11-12); right paramere medially constricted (Fig. 13); vesica with three main large lobes, without spiculum; one of the main lobes dentate and sclerotized; other two main lobes, membranous and divided into terminal lobes, surface of these lobes covered with microspinules, two of these terminal lobes apically dentate (Fig. 10).

FEMALE GENITALIA. Dorsal labiate plate large and prominent, with a large antero-medial projection, laterally with pointed and reinforced margins; sclerotized rings large, elongate-oval, separated, obliquely oriented; posterior wall with interramal sclerites, large and prominent interramal lobes (medially separated), large dorsal structure and median process (Fig. 89). 
COMMENTS. This species has been described from Iraq and here recorded from Iran for the first time. Collected on herbaceous host plants in meadow.

Stenotus Jakovlev, 1877

Stenotus binotatus (Fabricius, 1794)

Figs 1-2, 14-17, 83.

SYNONYMY: Stenotus paykulli (Turton, 1802); Stenotus sareptanus Jakovlev, 1877.

SPECIMENS EXAMINED. Iran, Guilan province: Totkabon;

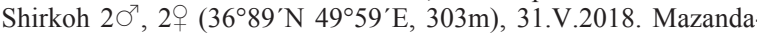
ran province: Ramsar $20^{\prime}, 2+\left(36^{\circ} 90^{\prime} \mathrm{N} 50^{\circ} 58^{\prime} \mathrm{E}, 580 \mathrm{~m}\right), 25$.VI.2018, M. Zamani, sweeping.
DIFFERENTIAL DIAGNOSIS. $S$. binotatus is easily distinguished from S. pallidus Reuter, 1904 and S. brevior Poppius, 1910 by its small size: 5.6-6ㅇ (in S. pallidus 6.36.7 ), presence of black markings on pronotum (in S. pallidus pronotum uniformly yellow; in $S$. brevior black markings indistinct), labium reaching metacoxa and often surpassing metacoxa (in S. pallidus short and not extending beyond the mesocoxa), $2^{\text {nd }}$ antennal segment $3 \mathrm{x}$ as long as $1^{\text {st }}$ antennal segment (4x in $S$. brevior), posterior margin of pronotum $2.3-2.5 \mathrm{x}$ as long as $1^{\text {st }}$ antennal segment ( $3 \mathrm{x}$ in $S$. brevior) [Poppius, 1905; Akramovskaya, Kerzhner, 1978].

This species has been reported from all zoogeographic regions. Namyatova et al. [2013] and Kim and Jung [2016]

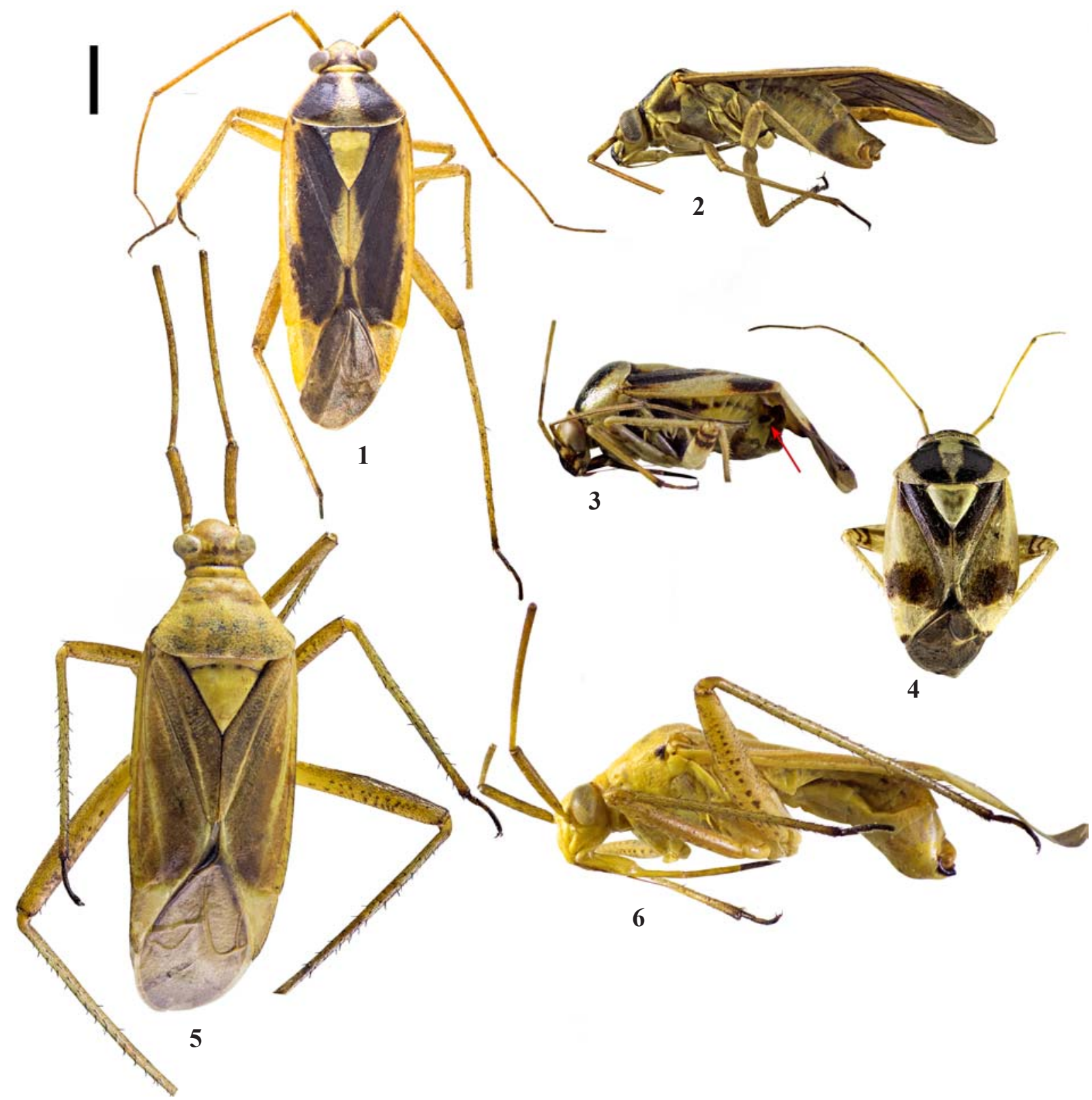

Figs 1-6. Habitus photographs of new record specimens (male): 1-2 - Stenotus binotatus; 3-4 - Cyphodema humbaba ; 5-6 Rauniella ishtar; 1, 4, 5- dorsal view; 2, 3,6 - lateral view. Scale bar: $1 \mathrm{~mm}$.

Рис. 1-6. Фотографии габитуса видов, впервые найденных в Иране (самцы): 1-2 - Stenotus binotatus; 3-4 - Cyphodema humbaba; 5-6-Rauniella ishtar; 1, 4, 5- сверху; 2, 3, 6- сбоку. Масштаб: 1 мм. 
provided detailed redescriptions quite recently. Here we have briefly redescribed the male and female genitalia as follow.

MALE GENITALIA. Left paramere sickle-shaped, sensory lobe strongly swollen and greatly expanded, apophysis prominent and widened, distally angulate, with flattened tip (Fig. 15); right paramere subapically with a depression, apophysis apically inclined upward, apex almost flattened (Figs 1617); vesica multilobed, membranous lobes apically spinose, medial lobe apically with concentration of enlarged spines (Fig. 14).
FEMALE GENITALIA. Dorsal labiate plate small; sclerotized rings small, elliptical, widely separated, oval, placed laterally and oriented vertically; posterior wall with interramal sclerites, spinulate interramal lobes (medially separated), dorsal structure and large median process (Fig. 83).

COMMENTS. Stenotus is composed of 53 species in the world (Schuh, 2002-2013). Eleven species are known in Palearctic region and only two species were previously recorded from Iran (including $S$. pallidus and S. brevior) [Wagner, 1968; Linnavuori, 2009]. Stenotus binotatus as a new record from Iran, was collected on Geramineous plants.
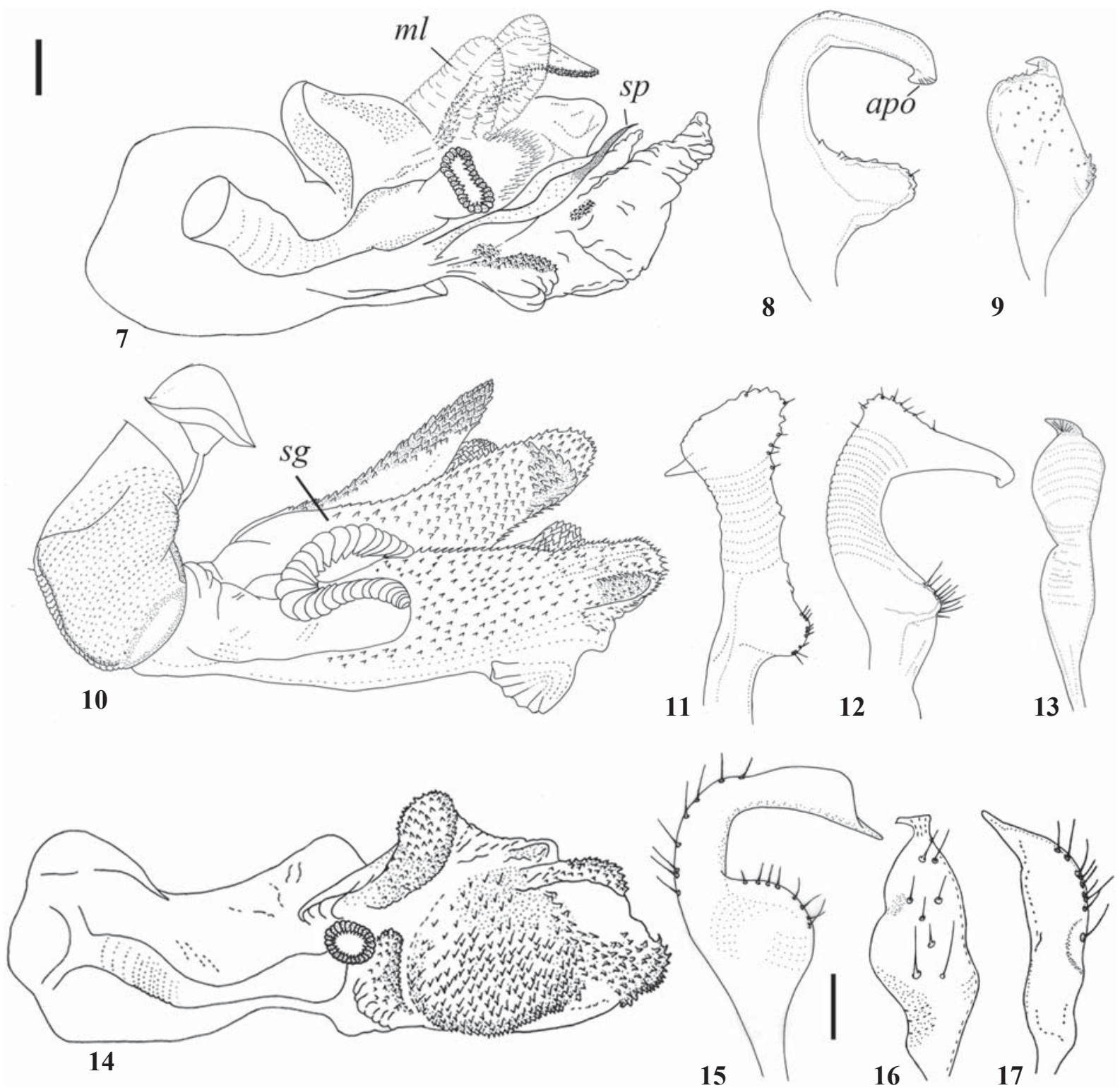

Figs 7-17. Male genitalia: 7-9 — Cyphodema humbaba; 10-13 - Rauniella ishtar; 14-17 - Stenotus binotatus; 7, 10, 14 - vesica; $8,11,12,15$ - left paramere; 9, 13, 16, 17 — right paramere; apo - apophysis; $m l$ - membranous lobe; $s g$ — secondary gonopore; $s p$ spiculum. Scale bar: $0.1 \mathrm{~mm}$ (7-13 and 14-17 same scale).

Рис. 7-17. Гениталии самцов: 7-9 - Cyphodema humbaba; 10-13 - Rauniella ishtar; 14-17 — Stenotus binotatus; 7, 10, 14 везика; 8, 11, 12, 15 - левая парамера; 9, 13, 16, 17 - правая парамера; аро — апофиз; $m l$ - мембранная лопасть; $s g$ - вторичный гонопор; $s p$ - спикулюм. Масштаб: 0.1 мм (7-13 и 14-17 в одном масштабе). 
Key to the Iranian Genera of Mirinae Subfamily

1. Ant like habitus (Fig. 18), collar completely obsolete, brachypterous (if macropterous, medially constricted), anterior segments of bulbous abdomen strongly constricted, lateral margins of abdomen abruptly upturned (tribe Herdoniini) Camponotidea

- Body not as above, collar distinct or posterior margin medially obsolete, macropterous, anterior segments of abdomen not constricted, lateral margins of abdomen not as above.

2. Body long and slender, head porrect, collar indistinct and medially not demarcated, antennal fossa stylate, lateral margins of pronotum carinate, $1^{\text {st }}$ segment of metatarsus longer than other segments (tribe Stenodemini) ......... 3

- Body oval or elongate-oval, head hypognathous, collar distinct, antennal fossa not stylate, lateral margins of pronotum not carinate (except Pantilius), $1^{\text {st }}$ segment of metatarsus shorter than others or subequal to $2^{\text {nd }}$ segment (except Stenotus) (tribe Mirini)

3. Vertex with a longitudinal median sulcus (Fig. 21), eyes almost touching anterior margin of pronotum
- Vertex with indistinct longitudinal median sulcus, eyes removed from pronotum ............. Leptopterna (Fig. 19)

4. Pronotum, scutellum and sometimes hemelytra distinctly and deeply punctate, vesica without spiculum

Stenodema (Fig. 21)

- Pronotum, scutellum and hemelytra impunctate or smoothly rugose, vesica with spiculum

5. Body large $\left(6-8.5 \bigcirc^{7} ; 8-9 \circ \mathrm{mm}\right), 1^{\text {st }}$ antennal segment much longer than head width across eyes ...

- Body small $\left(<5.5 \bigcirc^{\mathrm{T}} ; 5-6 \circ \mathrm{mm}\right), 1^{\mathrm{st}}$ antennal segment almost as long as or slightly longer than head width across eyes.

6. Clypeus visible from dorsal view, apex of frons smooth, metatibia with short adpressed setae, vesica with two spiculae (Fig. 30), sclerotized rings ovate and relatively small, dorsal structure small or obsolete (Figs 27-28) ....

Megaloceroea (Fig. 22)

- Clypeus not visible from dorsal view, apex of frons notched, metatibia with long erect setae, vesica with single spiculum (Fig. 26); sclerotized rings almost subrectangular and large, dorsal structure large (Fig. 25) ......... Notostria (Fig. 20)

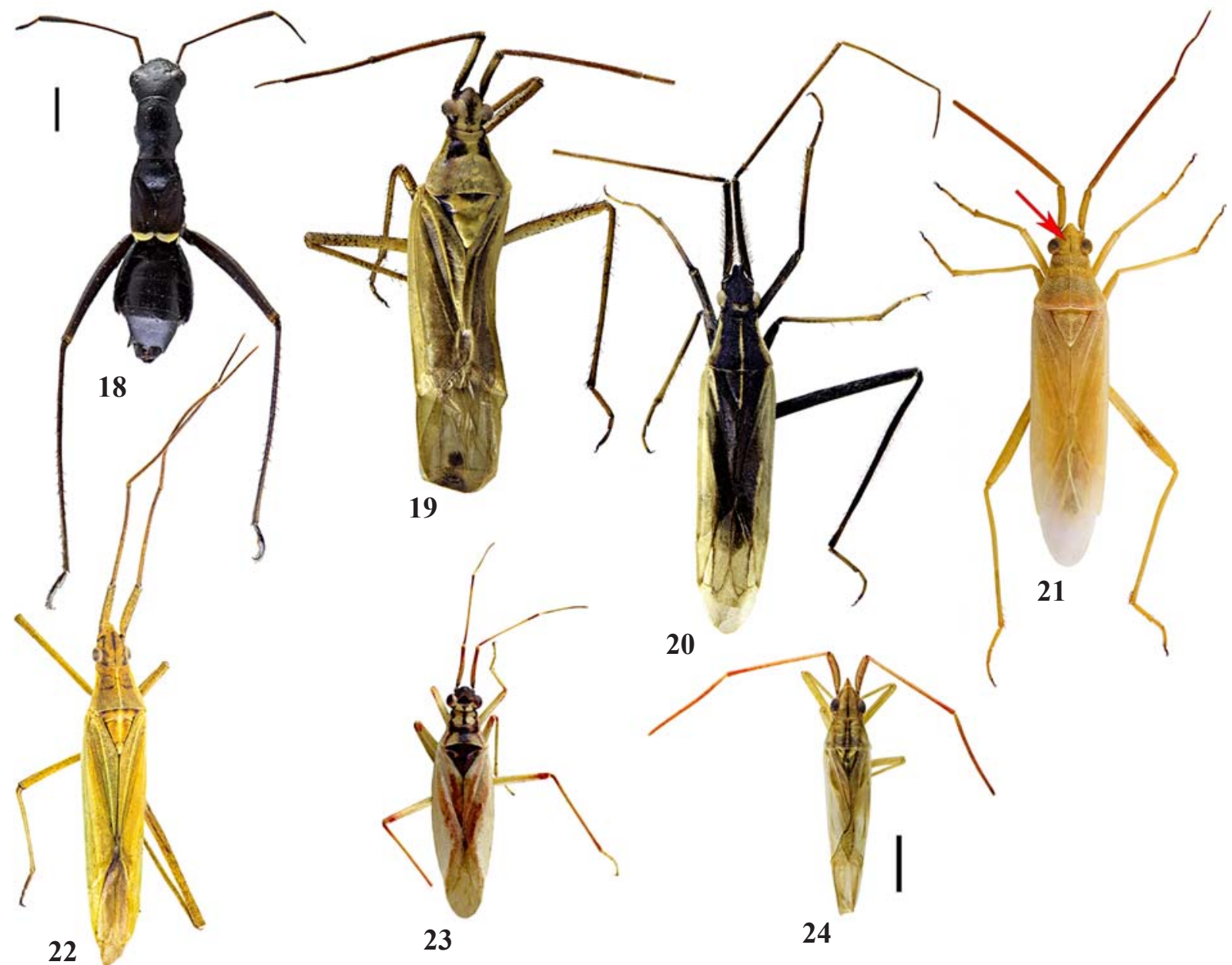

Figs 18-24. Dorsal habitus photographs of Stenodemini tribe (male): 18 - Camponotidea saundersi (Puton, 1874); 19 — Leptopterna putshkovi Vinokurov, 1982; 20 - Notostira poppiusi Reuter, 1911;21 - Stenodema turanica Reuter, 1904; 22 - Megaloceroea recticornis (Geoffroy, 1785); 23 - Teratocoris antennatus (Boheman, 1852); 24 — Trigonotylus caelestialium (Kirkaldy, 1902). Scale bar: $1 \mathrm{~mm}$ (1823 same scale).

Рис. 18-24. Габитус самцов трибы Stenodemini, сверху: 18 - Camponotidea saundersi (Puton, 1874); 19 - Leptopterna putshkovi Vinokurov, 1982; 20 - Notostira poppiusi Reuter, 1911; 21 - Stenodema turanica Reuter, 1904; 22 - Megaloceroea recticornis (Geoffroy, 1785); 23 - Teratocoris antennatus (Boheman, 1852); 24 - Trigonotylus caelestialium (Kirkaldy, 1902). Масштаб: 1 мм. (1823 в одном масштабе) 
7. Clypeus strongly projected, mandibular plates visible from dorsal view, apex of frons almost acute, eyes small, not globular; $1^{\text {st }}$ antennal segment proximally as thick as its apical part; vesica with single spiculum (Fig. 32); sclerotized rings small, dorsal labiate plate small and not developed (Fig. 29) Trigonotylus (Fig. 24)

- Clypeus blunt, mandibular plate not visible from dorsal view, apex of frons rounded, eyes large and globular; $1^{\text {st }}$ antennal segment proximally thicker than apically, vesica with 2 or 3 spiculae (Fig. 33); sclerotized rings obsolete, dorsal labiate plate well developed and strongly spinose (Fig. 31) Teratocoris (Fig. 23)

8. Dorsal surface punctuate or weakly punctate .............. 9

- Dorsal surface impunctate ............................................ 19

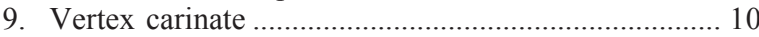

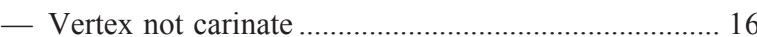

10. Tibia with golden spines ............................................. 11
- Tibia with dark spines

11. Dorsum covered with a mixture of whitish and blackish setae, sensory lobe of left paramere dentate, basally with a small projection and slender process (Fig. 72), vesica without spiculum, sclerotized rings of posterior wall large (Fig. 88) Taylorilygus (Fig. 34)

- Dorsum covered only with whitish setae, sensory lobe of left paramere smooth, basally without any projection or process, vesica with spiculum, sclerotized rings of posterior wall small

12. Body robust and oval, buccula broad, labium reaching mesocoxa (rarely metacoxa), $2^{\text {nd }}$ antennal segment $2.7-$ $3.5 \mathrm{x}$ as long as first, equals to or shorter than head width, left paramere with almost straight apophysis, distinctly elongated (Fig. 68); posterior wall with large, medially fused interramal lobes (Fig. 87) ... Agnocoris (Fig. 35)
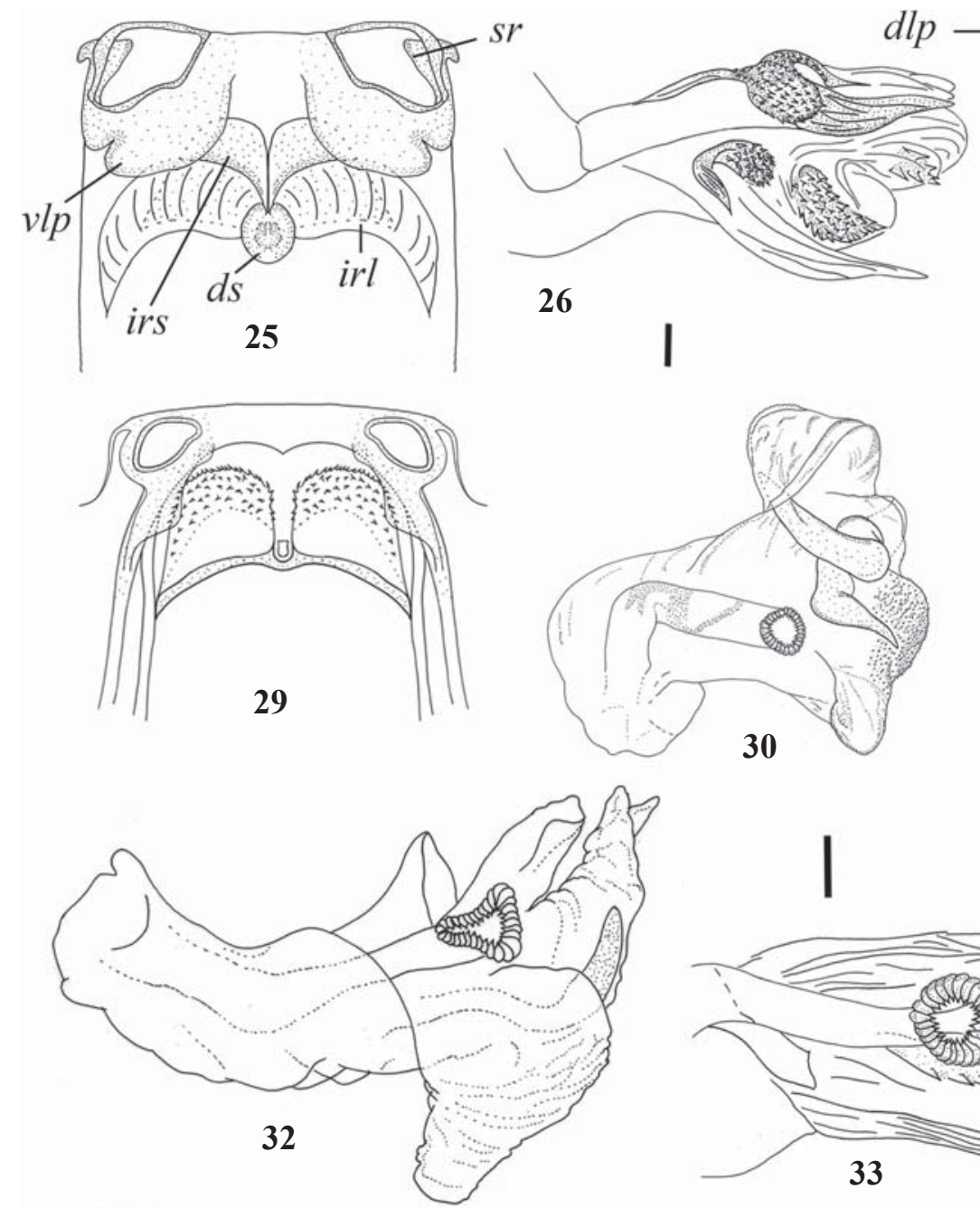

30

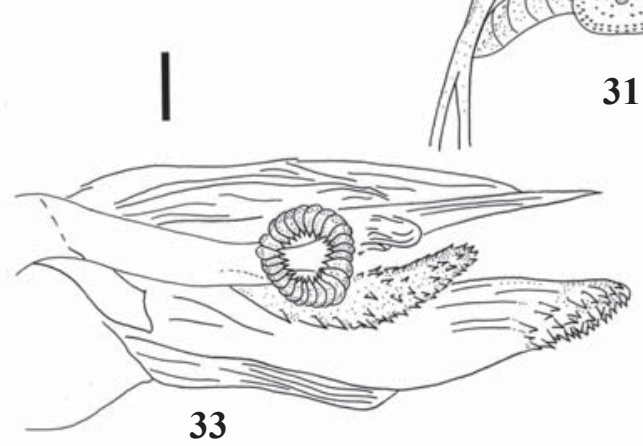

33

Figs 25-33. Male and female genitalia of Stenodemini tribe: 25-26 - Notostira poppiusi Reuter, 1911; 27-28, 30 - Megaloceroea recticornis (Geoffroy, 1785); 29,32 - Trigonotylus caelestialium (Kirkaldy, 1902); 31,33 - Teratocoris antennatus (Boheman, 1852); 25, 27-29, 31 - female genitalia; 26, 30, 32, 33 - vesica; $d l p$ - dorsal labiate plate; $d s$ - dorsal structure; irl — interramal lobes; irs interramal sclerites; $m p$ - median process; $s r$ — sclerotized ring; $v l p$ — ventral labiate plates. Scale bar: $0.1 \mathrm{~mm}(25-28,30 ; 29,31-33$ same scale); 25-26, 31, 33 - after Schwartz, 2008.

Рис. 25-33. Гениталии самцов и самок трибы Stenodemini: 25-26 - Notostira poppiusi Reuter, 1911; 27-28, 30 - Megaloceroea recticornis (Geoffroy, 1785); 29,32 - Trigonotylus caelestialium (Kirkaldy, 1902); 31,33 - Teratocoris antennatus (Boheman, 1852); 25, 27-29, 31 - гениталии самок; 26, 30, 32, 33 - везика; $d l p$ - дорсальные губные пластинки; $d s$ - дорсальная структура; irl интеррамальные лопасти; irs - интеррамальный склерит; $m p$ - медиальный отросток; $s r$ - склеротизованное кольцо; $v l p-$ вентральные губные пластинки. Масштаб: 0.1 мм (25-28, 30; 29, 31-33 в одном масштабе); 25-26, 31, 33 - по Schwartz, 2008. 
- Body elongate-oval, buccula narrow, labium reaching or surpassing metacoxa, $2^{\text {nd }}$ antennal segment $4.0-4.3 \mathrm{x}$ as long as first, much longer than head width, left paramere with curved apophysis; posterior wall with small, separated interramal lobes (Fig. 78) ............ Pinalitus (Fig. 36)

13. Vestiture composed of pale and black setae, vesica with sclerotized appendages; posterior wall without dorsal structure

... 14

- Vestiture uniformly whitish, vesica without sclerotized appendages; posterior wall with dorsal structure ...... 15

14. Pronotum coarsely punctuate, cuneus reddish to dark brown, basally with a black marking at inner part, apophysis of left paramere apically expanded (Fig. 59), vesica with two appendages (Fig. 58); outer margin of sclerotized rings rounded (Fig. 90) ..................... Charagochilus (Fig. 37)

- Pronotum finely punctuate, cuneus medially red (in species of the subgenus Poeciloscytus present in Iran), apophysis of left paramere apically not expanded (Fig. 61), vesica with more than two appendages (Fig. 60); outer margin of sclerotized rings acute (Fig. 85) ..... Polymerus (Fig. 38)

15. Body small $(3.5-5.2 \mathrm{~mm})$ and elongate-oval, collar narrow $(0.06 \mathrm{~mm})$, sensory lobe of left paramere with small tubercles (Fig. 69); lateral portion of dorsal structure narrow and reaching lateral edge of interramal sclerites, median process indistinct or absent (Fig. 86) ........ Orthops (Fig. 40)

- Body large (5.0-6.8 mm) and oval, collar broad (0.09$0.11 \mathrm{~mm}$ ), sensory lobe of left paramere dentate (Fig. 73); lateral portion of dorsal structure broad and not reaching lateral edge of interramal sclerites, median process distinct and small (Fig. 91) Lygus (Fig. 39)

16. Eyes very large, vertex not visible in lateral view, spines of hind tibia thick and distinctly longer than width of tibia, interocular width/width of eye $<10^{n}$... Lygidolon (Fig. 41)

- Eyes smaller, vertex visible in lateral view, spines of hind tibia narrow and shorter or as long as width of tibia, interocular width/width of eye $>10^{7}$

17

17. Pronotum and hemelytra uniformly black, $2^{\text {nd }}$ antennal segment clavate, sensory lobe of left paramere edentate,

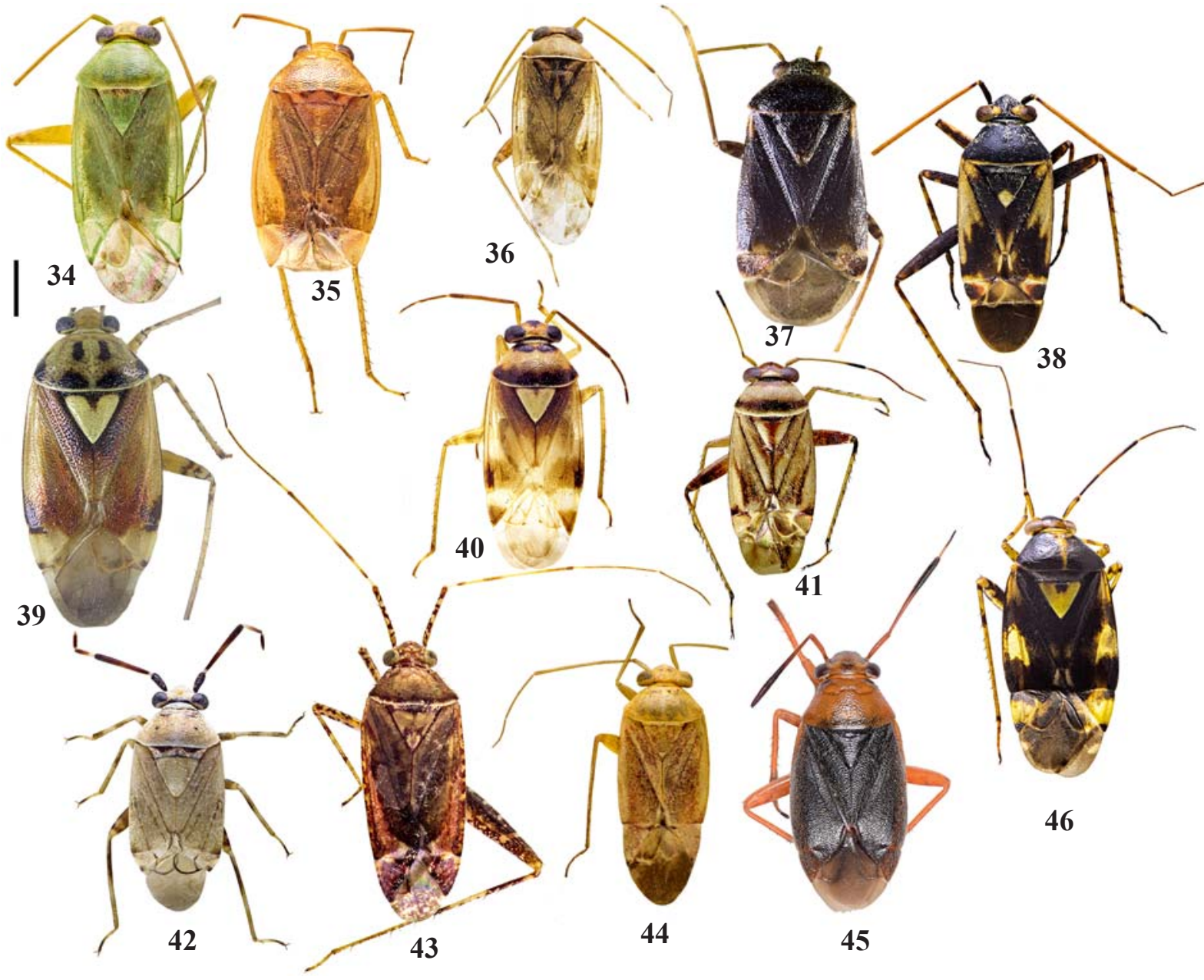

Figs 34-46. Dorsal habitus photographs of Mirini tribe (male): 34 - Taylorilygus apicalis (Fieber, 1861); 35 — Agnocoris reclairei (Wagner, 1949); 36 - Pinalitus cervinus (Herrich-Schaeffer, 1841); 37 - Charagochilus gyllenhali (Fallén, 1807); 38 - Polymerus cognatus (Fieber, 1858); 39 - Lygus pratensis (Linnaeus, 1758); 40 - Orthops kalmii (Linnaeus, 1758); 41 - Lygidolon eurystylioides (Wagner, 1957); 42 - Eurystylus bellevoyei (Reuter, 1879); 43 - Phytocoris scitulus Reuter, 1908; 44 — Dichrooscytus persicus Josifov, 1974; 45 - Capsus ater (Linnaeus, 1758); 46 - Liocoris tripustulatus (Fabricius, 1781). Scale bar: $1 \mathrm{~mm}$.

Рис. 34-46. Габитус самцов трибы Mirini: 34 - Taylorilygus apicalis (Fieber, 1861); 35 - Agnocoris reclairei (Wagner, 1949); 36 - Pinalitus cervinus (Herrich-Schaeffer, 1841); 37 - Charagochilus gyllenhali (Fallŭn, 1807); 38 - Polymerus cognatus (Fieber, 1858); 39 - Lygus pratensis (Linnaeus, 1758); 40 - Orthops kalmii (Linnaeus, 1758); 41 - Lygidolon eurystylioides (Wagner, 1957); 42 Eurystylus bellevoyei (Reuter, 1879); 43 - Phytocoris scitulus Reuter, 1908; 44 - Dichrooscytus persicus Josifov, 1974; 45 — Capsus ater (Linnaeus, 1758); 46 - Liocoris tripustulatus (Fabricius, 1781). Масштаб: 1 мм. 


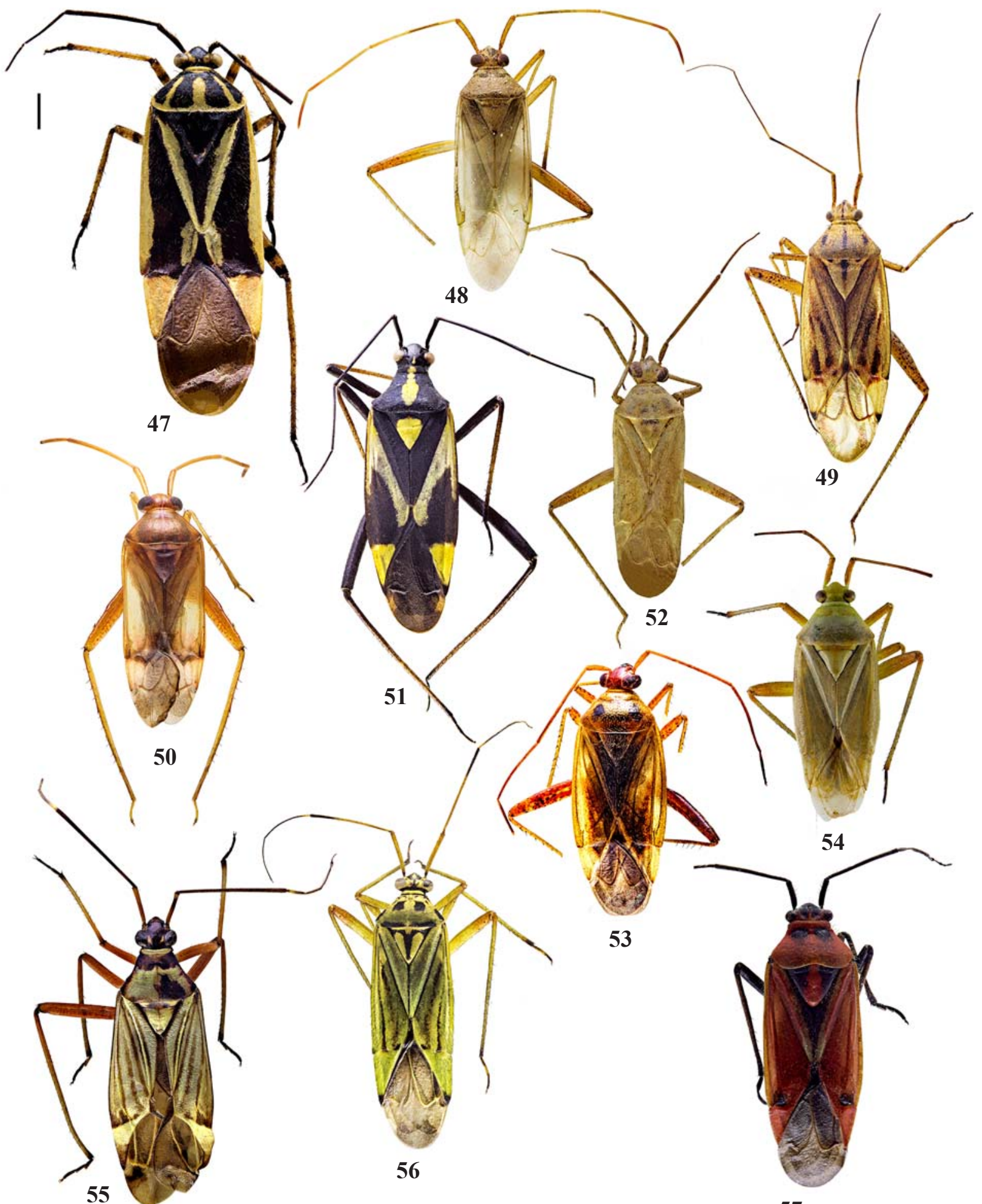

Figs 47-57. Dorsal habitus photographs of Mirini tribe (male): 47 - Brachycoleus steini Reuter, 1877; 48 — Creontiades pallidus (Rambur, 1839); 49 - Closterotomus costae (Reuter, 1888); 50 - Megacoelum sp.; 51 - Grypocoris golestanicus Heiss and Linnavuori, 2002; 52 - Reuterista villiersi (Wagner, 1968); 53 - Adelphocoris ticinensis (Meyer-Dür, 1843); 54 - Calocoris roseomaculatus roseomaculatus (De Geer, 1773); 55 - Miris persicus (Reuter 1876); 56 - Mermitelocerus schmidtii (Fieber, 1836); 57 — Horistus bimaculatus (Jakovlev, 1884). Scale bar: $1 \mathrm{~mm}$.

Рис. 47-57. Габитус самцов трибы Mirini: 47 - Brachycoleus steini Reuter, 1877; 48 - Creontiades pallidus (Rambur, 1839); 49 Closterotomus costae (Reuter, 1888); 50 - Megacoelum sp.; 51 - Grypocoris golestanicus Heiss and Linnavuori, 2002; 52 - Reuterista villiersi (Wagner, 1968); 53 - Adelphocoris ticinensis (Meyer-Dür, 1843); 54 - Calocoris roseomaculatus roseomaculatus (De Geer, 1773); 55 - Miris persicus (Reuter 1876); 56 - Mermitelocerus schmidtii (Fieber, 1836); 57 - Horistus bimaculatus (Jakovlev, 1884). Масштаб: 1 мм. 

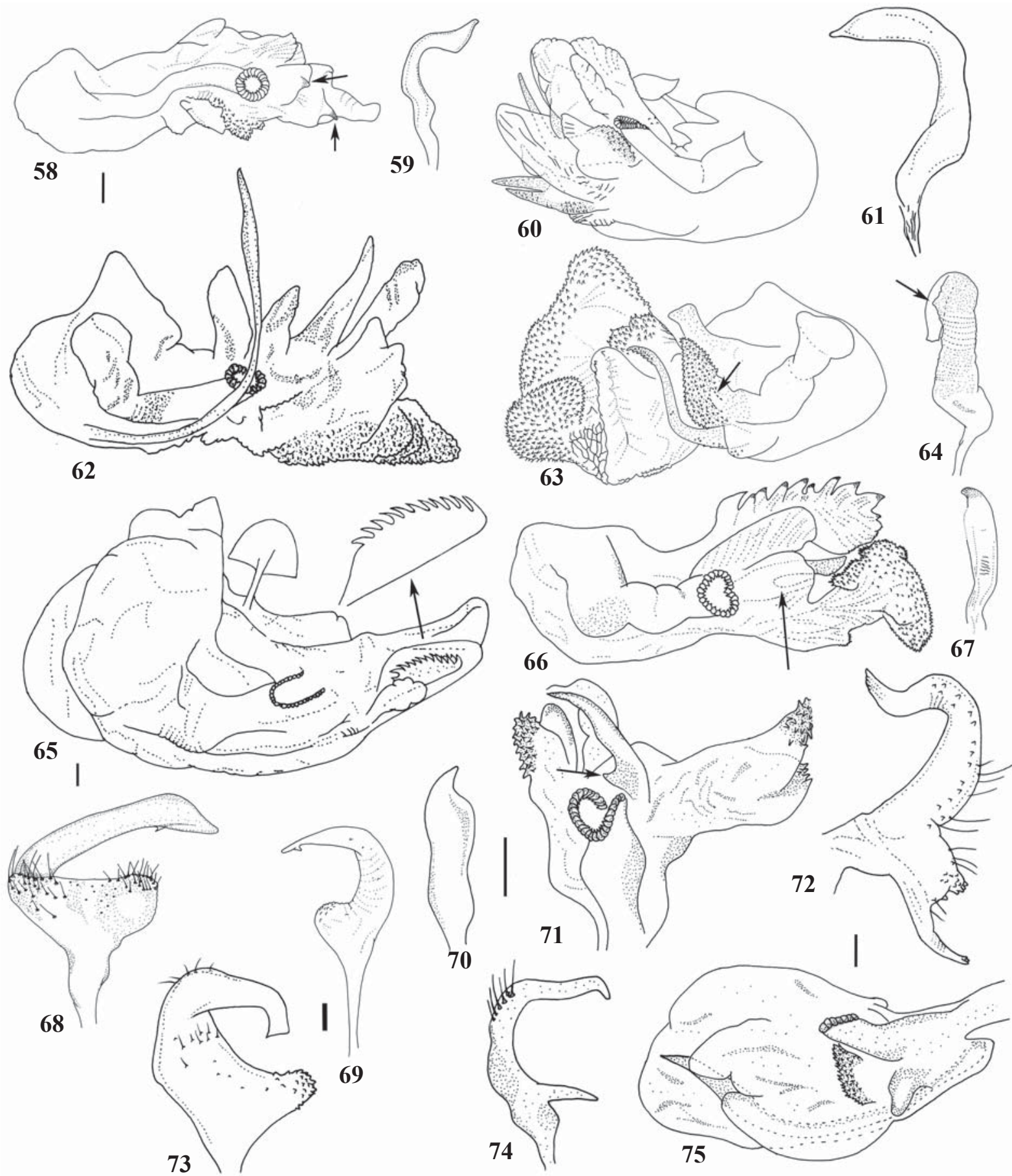

67

Figs 58-75. Male genitalia of Mirini tribe: 58-59 - Charagochilus gyllenhali (Fallén, 1807); 60-61 — Polymerus vulneratus (Panzer, 1806); 62 - Capsus wagneri Remane, 1950; 63 - Dichrooscytus persicus Josifov, 1974; 64 - Mermitelocerus schmidtii (Fieber, 1836); 65 - Adelphocoris vandalicus (Rossi, 1790); 66-67 - Closterotomus costae (Reuter, 1888); 68 - Agnocoris reclairei (Wagner, 1949); 69 - Orthops kalmii (Linnaeus, 1758); 70-71 — Reuterista villiersi (Wagner, 1968); 72 - Taylorilygus apicalis (Fieber, 1861); 73 - Lygus rugulipennis Poppius, 1911; 74-75 — Rhabdomiris striatellus (Fabricius, 1794); 58, 60, 62, 63, 65, 66, 71, 75 — vesica; 59, 61, 64, 68, 69, 72-74 - left paramere; 67,70 - right paramere. $(58-61,63,64,66,67 ; 62,65,68 ; 70,71 ; 72,74,75 ; 73,69$ — same scale). Scale bar: $0.1 \mathrm{~mm}$. (62 and 70, 71, 74, 75 - after Kim et al., 2015 and Rosenzweig, 1997, respectively).

Рис. 58-75. Гениталии самцов трибы Mirini: 558-59 - Charagochilus gyllenhali (Fallén, 1807); 60-61 - Polymerus vulneratus (Panzer, 1806); 62 - Capsus wagneri Remane, 1950; 63 - Dichrooscytus persicus Josifov, 1974; 64 - Mermitelocerus schmidtii (Fieber, 1836); 65 - Adelphocoris vandalicus (Rossi, 1790); 66-67 - Closterotomus costae (Reuter, 1888); 68 - Agnocoris reclairei (Wagner, 1949); 69 - Orthops kalmii (Linnaeus, 1758); 70-71 - Reuterista villiersi (Wagner, 1968); 72 - Taylorilygus apicalis (Fieber, 1861); 73 - Lygus rugulipennis Poppius, 1911; 74-75 — Rhabdomiris striatellus (Fabricius, 1794); 58, 60, 62, 63, 65, 66, 71, 75 - везика; 59, $61,64,68,69,72-74$ - левый парамер; 67, 70 - правый парамер. (58-61, 63, 64, 66, 67; 62, 65, 68; 70, 71; 72, 74, 75; 73, 69 — в одном масштабе). Масштаб: 0.1 мм. (62 and 70, 71, 74, 75 - по Kim et al., 2015 и Rosenzweig, 1997, соответственно). 
spiculum of conjunctiva long and narrow (Fig. 62) ....... Capsus (Fig. 45)

- Pronotum and hemelytra yellowish and black, $2^{\text {nd }}$ antennal segment cylindrical, sensory lobe of left paramere dentate, spiculum of vesica short and broad

18

18. Tibia with pale spines, pygophore with large and prominent denticle on the left side (Fig. 3), basal margin of right paramere dentate (Fig. 9); dorsal structure of posterior wall broadly rounded, disk-shaped and spinulate (Fig. 81), dorsal margin of second valvula apically with sparse teeth (Fig. 94) Cyphodema (Fig. 3-4)

- Tibia with dark spines, pygophore without denticle, basal margin of right paramere edentate, dorsal structure of posterior wall not as above; dorsal margin of second valvula with a seri of dense teeth at apex (Fig. 96) ........ Liocoris (Fig. 41)

19. Hemelytra greenish, often mottled with small reddish dots, posterior margin of vertex laterally carinate, vesica with short appendage (Fig. 63); margins of sclerotized rings medially form connecting bar, interramal sclerites medially separated (Fig. 77) .... Dichrooscytus (Fig. 44)

- Coloration of hemelytra variable, posterior margin of vertex not carinate, vesica without appendage; margins of sclerotized rings not united at middle, interramal sclerites partly or completely fused

20

20. First segment of metatarsus distinctly longer than other segments, genital opening oriented posteriorly, apophysis of left paramere with flattened tip (Fig. 15); ventral and dorsal margins of second valvula without teeth (Fig. 95) Stenotus (Figs 2-3)

- First segment of metatarsus shorter than other segments, genital opening oriented dorsally, apophysis of left paramere not as above, ventral and dorsal margins of second valvula with a few teeth (except Brachycoleus in its dorsal margin)

.... 21

21. Hemelytra with regular, dense, orange to red or irregular dark mottling, rarely uniformly pale, $1^{\text {st }}$ antennal segment longer than head width, antenna usually longer than (or as long as) body length, metafemora long, usually reaching beyond apex of abdomen ............... Phytocoris (Fig. 43)

- Hemelytra not as above, $1^{\text {st }}$ antennal segment as long as or shorter than head width, antenna shorter than body length, metafemora not reaching apex of abdomen ............... 22

22. Vestiture mixed, consisting of short adpressed black and whitish setae, or dorsum covered with long and semierect setae (in Brachycoleus)

- Vestiture homogenous, blackish or whitish, if mixed, body uniformly pale greenish to brownish (in Rauniella) ... 27

23 . Body small $(3-4 \mathrm{~mm})$, remarkably robust and oval, $1^{\text {st }}$ antennal segment tumid, $2^{\text {nd }}$ antennal segment clavate.. Eurystylus (Fig. 42)

- Body larger $(>5 \mathrm{~mm})$, slender and elongated, $1^{\text {st }}$ antennal segment cylindrical, $2^{\text {nd }}$ antennal segment cylindrical or weakly incrassate distally (in Pantilius)

24

24 . Body robust, frons markedly projecting over clypeus 25

- Body delicate, frons slightly convex ........................... 26

25 . Body covered with short and adpressed setae, vertex with distinct median longitudinal sulcus, $2^{\text {nd }}$ antennal segment weakly incrassate distally, lateral margins of pronotum carinate

Pantilius

- Body covered with long and semierect setae, vertex without median longitudinal sulcus, $2^{\text {nd }}$ antennal segment cylindrical, lateral margin of pronotum not carinate ....

Brachycoleus (Fig. 47)

26. Labium reaching middle of mesosternum or mesocoxa, interocular width/width of eye $<1 \sigma^{7}$, cuneus stramine- ous to greenish, apophysis of right paramere acute at apex (Fig. 70), process of spiculum short and reduced to basal tooth (Fig. 71), sometimes inconspicuous ..........

Reuterista (Fig. 52)

- Labium reaching metacoxa (rarely mesocoxa), interocular width/width of eye $>10^{7}$, cuneus tinged with dark red basally, apex blackish; apophysis of right paramere blunt at apex (Fig. 67), process of spiculum long (Fig. 66) ...

Closterotomus (Fig. 49)

27. Vertex with longitudinal shallow or deep midline sulcus, femora with a regular seri of spines, apophysis of left paramere ending with acute process, dorsal structure of posterior wall small

28

- Vertex without midline sulcus, femora apically with a few spines, apophysis of left paramere not as above (except Grypocoris fieberi); dorsal structure of posterior wall

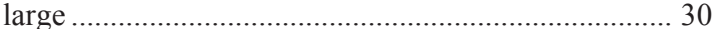

28. Vertex with deep sulcus, tibiae with pale spines, pygophore without denticle, vesica without spiculum; dorsal labiate plate with anteromedial projection (Fig. 82) ..... Creontiades (Fig. 48)

- Vertex with shallow sulcus, tibia with black spines, pygophore with denticle, vesica with spiculum; dorsal labiate plate without antero-medial projection ..................... 29

29. Vestiture adpressed, spiculum comb-shaped (Fig. 65); dorsal labiate plate developed, sclerotized rings almost subcontiguous (Fig. 76) ............. Adelphocoris (Fig. 53)

- Vestiture semierect, spiculum sickle-shaped; dorsal labiate plate reduced, sclerotized rings distinctly separated (Fig. 80) .................................... Megacoelum (Fig. 50)

30. Body covered with erect to semierect setae, eyes small, interocular width/width of eye $>2$..... Horistus (Fig. 57)

- Body covered with adpressed setae, eyes large, interocular width/width of eye $<2$

31

31. Secondary gonopore like an inverted saddle (Fig. 10); dorsal labiate plate with antero-medial projection (e.g. Figs 82, 89) ........................................................ 32

- Secondary gonopore variable; dorsal labiate plate without antero-medial projection ........................................... 33

32. Pubescence mixed of whitish and black, coloration uniformly greenish to brownish, apophysis of left paramere subapically with a large dentate projection (Figs 11-12)

Rauniella (Figs 5-6)

- Pubescence whitish, coloration black and yellow, apophysis of left paramere subapically without projection . Grypocoris (Fig. 51)

33. Pubescence whitish, sensory lobe of left paramere with long acute process (Fig. 74), vesica with long spiculum (Fig. 75) Rhabdomiris

- Pubescence black, sensory lobe of left paramere without process, vesica without spiculum ............................... 34

34. Coloration black and yellow or red, antenna almost as long as body length, medial vein between the radial and cubital veins present on corium.............. Miris (Fig. 55)

- Coloration yellowish to greenish, antenna shorter than body length, medial vein between the radial and cubital veins absent on corium .............................................. 35

35. Labium reaching mesocoxa, $1^{\text {st }}$ antennal segment $2.5-3 \mathrm{x}$ longer than interocular distance, lateral margin of left paramere with a projection (Fig. 64); dorsal labiate plate membranous, sclerotized rings oval (Fig. 79), first valvula broadly triangular, apically acute (Fig. 92)

Mermitelocerus (Fig. 56)

- Labium reaching metacoxa, $1^{\text {st }}$ antennal segment 1.4 1.6x longer than interocular distance, lateral margin of left paramere without projection; dorsal labiate plate 

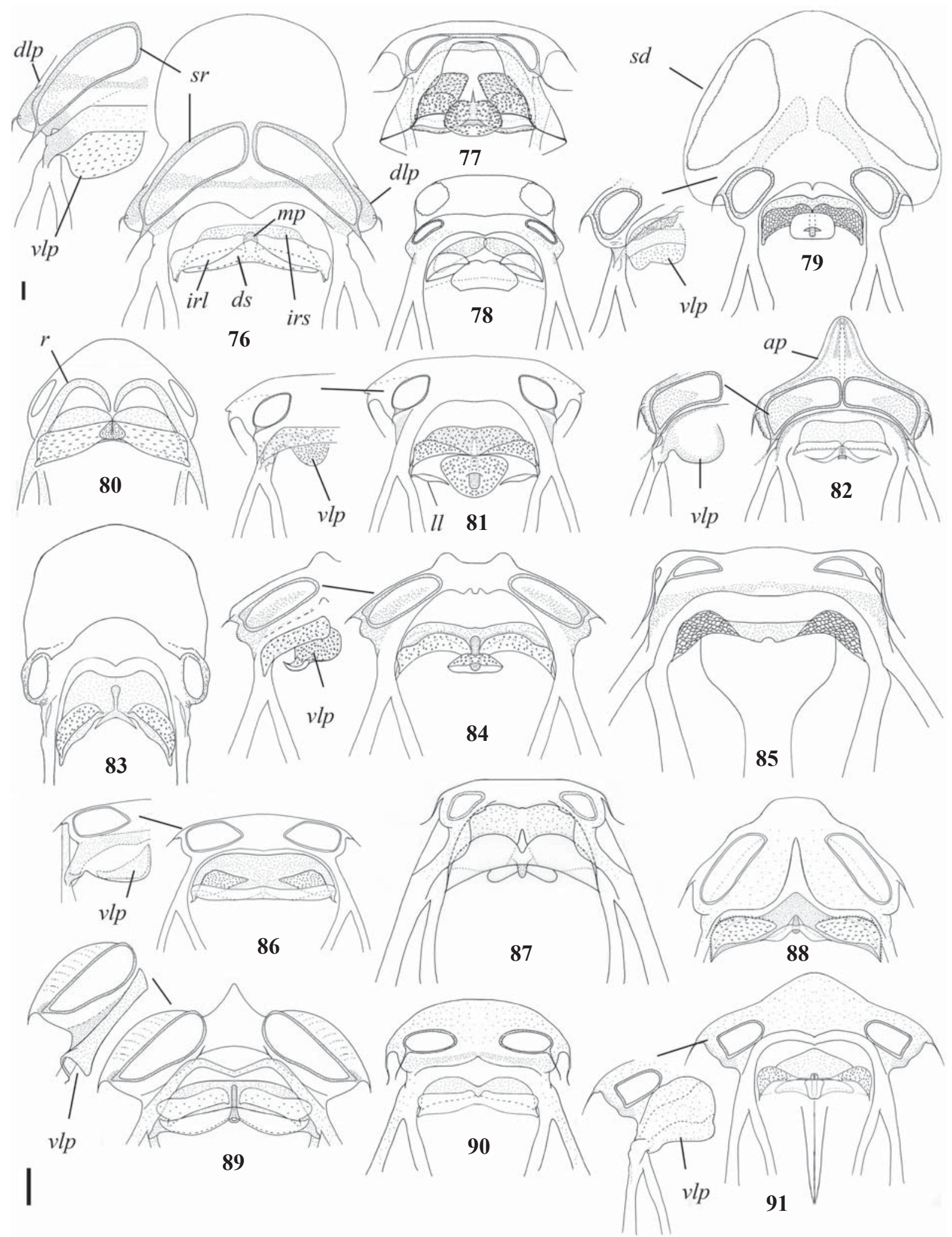

Figs 76-91. Female genitalia of Mirini tribe: Sclerotized ring and posterior wall of bursa copulatrix with their associated structures: 76 - Adelphocoris vandalicus (Rossi, 1790); 77 - Dichrooscytus persicus Josifov, 1974; 78 — Pinalitus cervinus (Herrich-Schaeffer, 1841); 79 - Mermitelocerus schmidtii (Fieber, 1836); 80 - Megacoelum sp.; 81 - Cyphodema humbaba Linnavuori, 1984; 82 — Creontiades pallidus (Rambur, 1839); 83 - Stenotus binotatus (Fabricius, 1794); 84 — Calocoris roseomaculatus roseomaculatus (De Geer, 1773); 85 - Polymerus vulneratus (Panzer, 1806); 86 - Orthops kalmii (Linnaeus, 1758); 87 - Agnocoris reclairei (Wagner, 1949); 88 - Taylorilygus apicalis (Fieber, 1861); 89 - Rauniella ishtar (Linnavuori, 1984); 90 - Charagochilus gyllenhali (Fallén, 1807); 
moderately sclerotized, sclerotized rings elongate-oval (Fig. 84), first valvula blade shaped (Fig. 93)

Calocoris (Fig. 54)

\section{Discussion}

In the current study, a comprehensive identification key was compiled for genera belonging to three tribes of the Mirinae subfamily found in Iran, and the number of valid recorded genera for Iranian fauna increased from 34 to 35 . In this key, different characters were used for categorization of tribes or genera ranks. Schwartz [2008] considered characters including: body form, head structure, longitudinal sulcus of the frons, lateral margin of the pronotum, pretarsal and metatarsal structure, dorsal surface structure, male and female genitalic structure as the most useful in recognizing intertribal relationship within the Mirinae. Rosenzweig [2001] emphasized that the most reliable taxonomic characters in the Mirinae are male genitalia (vesica and left paramere), female genitalia (location and shape of interramal lobes, presence and symmetry of the median process) and the

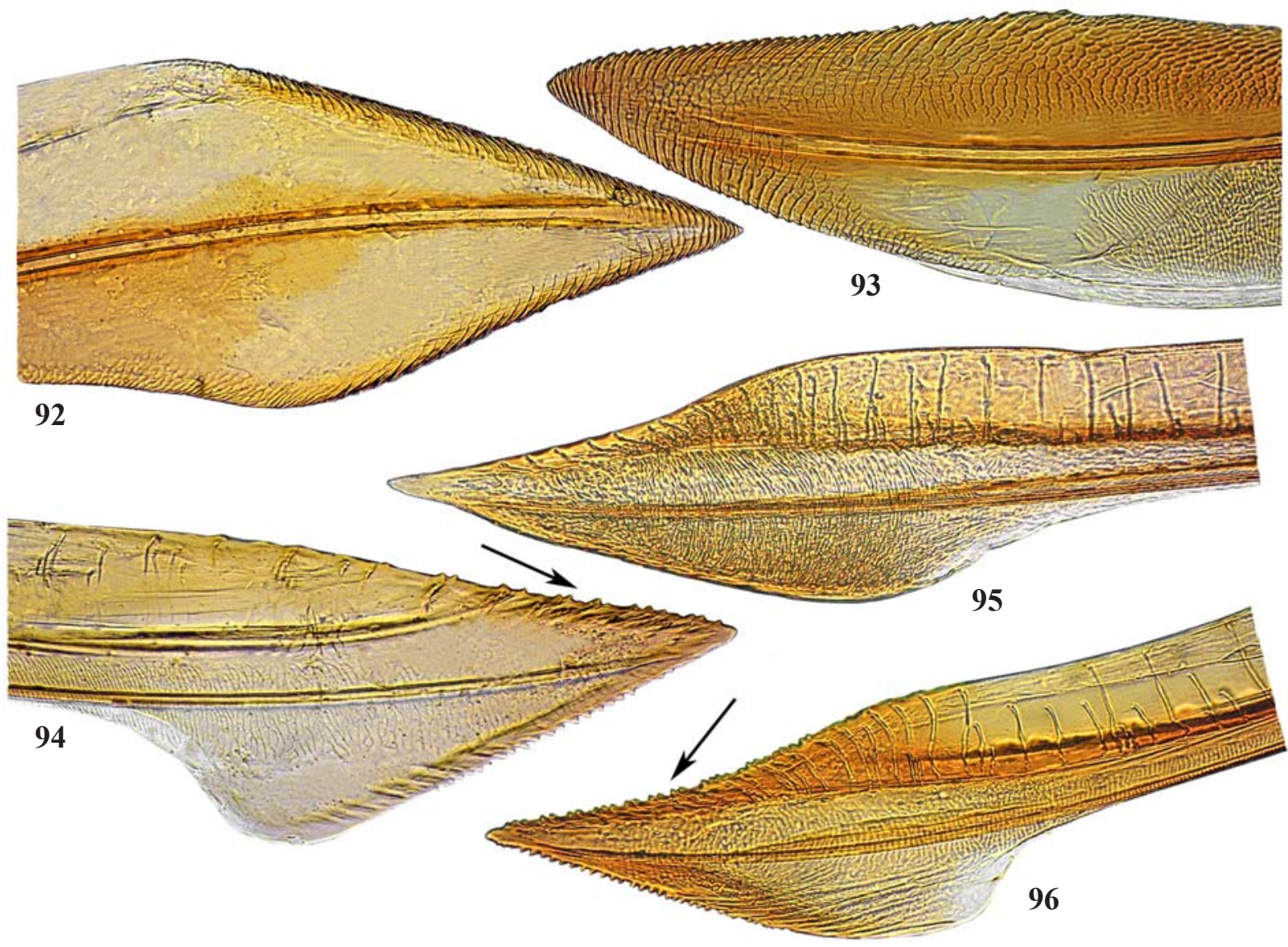

Figs 92-96. First and second valvula of ovipositor of female genitalia: 92 - Mermitelocerus schmidtii (Fieber, 1836); 93 — Calocoris roseomaculatus roseomaculatus (De Geer, 1773); 94 - Cyphodema humbaba Linnavuori, 1984; 95 - Stenotus binotatus (Fabricius, 1794); 96 - Liocoris tripustulatus (Fabricius, 1781); 92, 93 - first valvula; 94-96 - second valvula.

Рис. 92-96. Наружные и внутренние створки яйцеклада самки: 92 - Mermitelocerus schmidtii (Fieber, 1836); 93 - Calocoris roseomaculatus roseomaculatus (De Geer, 1773); 94 - Cyphodema humbaba Linnavuori, 1984; 95 — Stenotus binotatus (Fabricius, 1794); 96 - Liocoris tripustulatus (Fabricius, 1781); 92, 93 - наружные створки; 94-96 - внутренние створки.

91 - Lygus gemellatus (Herrich-Schaeffer, 1835). ap — antero-medial projection of parieto-vaginal ring; dlp — dorsal labiate plate; irl — interramal lobes; irs — interramal sclerites; $l l$ - lateral lobes; $m p$ - median process; $r$ — ramus; $s d$ - seminal depository; $s r$ - sclerotized ring; $v l p$ — ventral labiates plate. $(76,77,79-83,89,91 ; 78,84-88,90$ same scale). Scale bar: $0.1 \mathrm{~mm}$.

Рис. 76-91. Гениталии самок трибы Mirini. Склеротизованное кольцо и задняя стенка копулятивной сумки и связанные с ними структуры: 76 - Adelphocoris vandalicus (Rossi, 1790); 77 - Dichrooscytus persicus Josifov, 1974; 78 - Pinalitus cervinus (HerrichSchaeffer, 1841); 79 - Mermitelocerus schmidtii (Fieber, 1836); 80 - Megacoelum sp.; 81 - Cyphodema humbaba Linnavuori, 1984; 82 - Creontiades pallidus (Rambur, 1839); 83 - Stenotus binotatus (Fabricius, 1794); 84 - Calocoris roseomaculatus roseomaculatus (De Geer, 1773); 85 - Polymerus vulneratus (Panzer, 1806); 86 - Orthops kalmii (Linnaeus, 1758); 87 - Agnocoris reclairei (Wagner, 1949); 88 - Taylorilygus apicalis (Fieber, 1861); 89 - Rauniella ishtar (Linnavuori, 1984); 90 - Charagochilus gyllenhali (Fallén, 1807); 91 — Lygus gemellatus (Herrich-Schaeffer, 1835). ap — антеро-медиальное проекция; dlp — дорсальная губная платинка; irl — интеррамальная лопасть; $i r s$ - интеррамальный склерит; $l l$ - латеральные лопасти; $m p$ - медиальный отросток; $r$ - рамус; $s d$ семяприемник; $s r$ - склеротизованное кольцо; $v l p$ — вентральная губная пластинка. $(76,77,79-83,89,91 ; 78,84-88,90$ в одном масштабе). Масштаб: 0.1 мм. 
color and type of vestiture. Most of the above-mentioned characters were used for compiling the key.

Among three tribes studied in this research, Mirini is the most species-rich tribe which includes many genera complexes. For example Calocoris and Closterotomus complexes show similarities in their external appearance and genitalia which make them difficult to identify [Rosenzweig, 1997; Cherot, Malipatil, 2016]. For development of a generic taxonomical key consideration of genera complexes is an essential task. Rosenzweig [2001] reclassified the genera belonging to Mirina subtribe based on morphology by considering genera complexes and their similarities. Therefore in this study, characteristics of genera complexes used by different authors [e.g. Kelton 1955; Stonedahl, 1988; Rosenzweig, 2001] were mainly considered in the current key. Genera were mostly separated by their positions on their relevant complexes and coupled with their most similar genera on the base of morphological characters similarities. The aim of the present contribution was to enable more people to identify Mirinae specimens of the Miridae family collected from Iran or adjacent countries.

Acknowledgements. Authors wish to thank Saadi Mohammdi (Educated Msc student, University of Guilan) for his contribution in collecting of specimens for these study including two new records, Barış Çerçi (Hacettepe University, Turkey) for providing habitus photo of Capsus ater, and Dr. M.D. Schwartz (AMNH) for his advices regarding to definition of some scientific terms. The anonymous reviewers are also appreciated for their constructive comments.

\section{References}

Akramovskaya E.G., Kerzhner I.M. 1978. [Two new species of the genus Stenotus Jak. (Heteroptera, Miridae) from the USSR] // Entomologicheskoe Obozrenie. Vol.57. No.4. P.825-827 [in Russian; English translation: Entomological Review. Vol.57. P.567-569].

Aukema B., Rieger C. 1999. Catalogue of the Heteroptera of the Palaearctic Region. Vol.3. Netherlands Entomological Society, Ponsen and Looijen, Wageningen, 577 pp.

Aukema B., Rieger C., Rabitsch W. 2013. Catalogue of the Heteroptera of the Palaearctic Region. Vol.6. Supplement. Ponsen and Looijen, Wageningen: Netherlands Entomological Society. 628 pp.

Carvalho J.C.M. 1955. Keys to the genera of Miridae of the world (Hemiptera) // Boletim de Museu paraense 'Emilio Goeldi'. Vol.11. No.2. P.5-151.

Cassis G., Schuh R.T. 2012. Systematics, biodiversity, biogeography and host association of the Miridae (Insecta: Hemiptera: Heteroptera: Cimicomorpha) // The Annual Review of Entomology. Vol.57. P.377-404.

Cherot F., Malipatil M.B. 2016. A review of Adelphocoris-Creontiades-Megacoelum complexes (Hemiptera: Heteroptera: Miridae: Mirini), with description of two new genera and four new species // Zootaxa. Vol.4126. No.2. P.151-206.

Ghahari H., Cherot F. 2014. An annotated catalog of the Iranian Miridae (Hemiptera: Heteroptera: Cimicomorpha) // Zootaxa. Vol.3845. No.1. P.1-101.

Hosseini R. 2014. A study on the genus Orthops Fieber (Hemiptera: Miridae: Mirinae) in Iran // Arthropods. Vol.3. No.1. P.57-69.

Hosseini R. 2015. Redescription of the plant bug Miris persicus Reuter (Hemiptera: Heteroptera: Miridae: Mirinae) // Entomologica Americana. Vol.121. No.1. P.18-22.

Hosseini R. 2016. A review on the genus Brachycoleus (Hemiptera, Miridae) with identification key to the species found in Iran // Vestnik Zoologii. Vol.50. No.2. P.105-110.

Kelton L.A. 1955. Genera and subgenera of the Lygus complex
(Hemiptera: Miridae) // The Canadian Entomologist. Vol.87. No.7. P.277-301.

Kerzhner I.M. 1964. [Family Miridae (Capsidae) plant bugs] // G.Ya. Bey-Bienko (ed.). Opredelitel nasekomykh evropeyskoy chaste SSR. Leningrad. Vol.1. P.700-765 [in Russian].

Kim J., Jung S. 2016. Taxonomic review of the genus Stenotus Jakovlev (Hemiptera: Heteroptera: Miridae) from the Korean Peninsula // Journal of Asia-Pacific Biodiversity. Vol.9. No.1. P.29-33.

Kim J., Park H., Heiss E., Jung S. 2015. A new species of the genus Capsus Fabricius (Hemiptera: Heteroptera: Miridae: Mirinae) from the Korean Peninsula, with a key to the Korean Capsus species // Zootaxa. Vol.3905. No.4. P.585-592.

Konstantinov F.V. 2003. Male genitalia in Miridae (Heteroptera) and their significance for supra-generic classification of the family. Part I: general review, Isometopinae and Psallopinae // Belgian Journal of Entomology. Vol.5. P.3-36.

Linnavuori R.E. 1999. On the genus Phytocoris Fallén (Heteroptera, Miridae, Mirinae) in Iran with remarks on species of the adjacent countries. Part I // Acta Universitatis Carolinae Biologica. Vol.43. Nos3/4. P.163-193.

Linnavuori R.E. 2006. Studies on the Miridae (Insecta: Heteroptera) of Guilan and the adjacent provinces in northern Iran. I. Description of new species // Acta Universitatis Carolinae. Vol.49. Nos3-4. P.219-243.

Linnavuori R.E. 2009. Studies on the Nepomorpha, Gerromorpha, Leptopodomorpha and Miridae excluding Phylini (Hemiptera: Heteroptera) of Khozestan and the adjacent provinces of Iran // Acta Entomologica Musei Nationalis pragae. Vol.49. No.1. P.132.

Namyatova A.A., Schwartz M.D., Cassis G. 2013. First record of the genus Stenotus Jakovlev from Australia, with two new species, and a list of mirine species from Witchelina Nature Reserve (Insecta: Heteroptera: Miridae: Mirinae: Mirini) // Journal of Natural History. Vol.47. Nos13-14. P.987-1008.

Poppius B. 1905. 12. Hemiptera. 4. Miridae, Anthocoridae, Termatophylidae, Microphysidae und Nabidae. Wissenschaftliche Ergebnisse der schwedischen zoologischen Expedition nach dem Kilimandjaro, dem Meru und den umgebenden Massaisteppen Deutsch-Östafrikas, 1906. P.25-60.

Rosenzweig V.Ye. 1997. Revised classification of the Calocoris complex and related genera (Heteroptera: Miridae) // Zoosystematica Russica. Vol.6. Nos1/2. P.139-169.

Rosenzweig V.Ye. 2001. Synoptic of the Palearctic genera of Mirina (Heteroptera: Miridae) // Zoosystematica Russica. Vol.6. Nos12. P.367-383.

Schuh R.T., Slater J.A. 1995. True bugs of the world (Hemiptera: Heteroptera). Classification and Natural History. Ithaca, New York: Cornell University. Press. 336 pp.

Schuh R.T. 2002-2013. On-line systematic catalog of plant bugs (Insecta: Heteroptera: Miridae). The American Museum of Natural History, New York, Available from http://research.amnh.org/pbi/ catalog.

Schwartz M.D. 2008. Revision of the Stenodemini with a Review of the Included Genera (Hemiptera: Heteroptera: Miridae: Mirinae) // Proceedings of the Entomological Society of Washington. Vol.110. No.4. P.1111-1201.

Scudder G.G., Schwartz M.D. 2012. Two new species of Trigonotylus (Hemiptera: Heteroptera: Miridae: Stenodemini) from western Canada and northwestern United States // Zootaxa. Vol.3714. P.51-58.

Slater J.A. 1950. An investigation of the female genitalia as taxonomic characters in the Miridae (Hemiptera) // Iowa State College Journal of Science. Vol.25. P.1-81.

Stonedahl G.M. 1988. Revision of the Phytocoris Fallen (Heteroptera, Miridae) for Western North America // Bulletin of the American Museum of Natural History. Vol.188. No.1. P.1-257.

Wagner E. 1968. Contribution a la fauna de l'Iran. 7. Hemipteres: Heteropters (pro parte) // Annales de la Societe Entomologique de France (N.S). Vol.4. P.437-453.

Wagner E. 1974. Die Miridae Hahn, 1831 des Mittelmeerraumes und der Makaronesischen Inseln (Hemiptera, Heteroptera), Teil I // Entomol. Abh. Staatl. Mus. Tierk. Dresden. Bd.37. S.1-484.

Wagner E., Weber H.H. 1964. Heteropteres Miridae // Faune de France. Vol.67. P.1-590.

Wheeler A.G. 2001. Biology of the plant bugs (Hemiptera: Miridae) // Ithaca and London: Cornell University Press. 507 pp. 